\title{
Dependence of Consumption Volume on the Labor-Force in One-Productive Models of Economic Dynamics
}

\author{
Sabir Isa Hamidov \\ Baku State University, AZ1021, Baku Azerbaijan.
}

Received: January 22, 2015 / Accepted: February 25, 2015 / Published: March 25, 2015.

\begin{abstract}
In the work one-productive model of economic dynamics is considered. The dependence of the consumption volume on the labor-force involved in production is investigated the case when production function coincides with the Cobb-Douglas function or function with constant elasticity of substitution (CES) is studied.
\end{abstract}

Keywords: One-productive models, production function, Cobb-Douglas function, function with constant elasticity of substitution (CES).

In this paper we study the problem of dependence of the volume of consumption of the labor force in the framework of a simple one-commodity model of economic dynamics. Consider two adjacent points in time moments $\bar{t}, t$. Then the model may be given by the relations

$$
F(\widetilde{K}, \widetilde{L})=I+C, c=\omega L, K=v \widetilde{K}+I .
$$

Here $\widetilde{K}$ and $\widetilde{L}$ are amount of funds and the size of the labor force in the current time $\bar{t} ; K$ и $L$ amount of funds and the size of the labor force in the next time $t$ (time is taken discrete); $I$ - investment, $C$ is a consumption, $\omega$ - Specific consumption (wage rate); $F$ - product function, $v$ - funds safety coefficient. It is assumed that the volume of consumption $C$ produced by the labor force $\widetilde{L}$ at the time $t$, advanced by the labor force $L$ involved in this moment.

In the study of the described models often is assumed that given constant rate of the accumulation $S$, which is independent of the labor force $L$.

Corresponding authot: Sabir Isa Hamidov, Ph.D., ass.professor, research field: mathematical economics. E-mail: sabir818@yahoo.com.
Let $L=\rho \widetilde{L}$, where $\rho$ is a labor force growth. Then

$$
C(L)=(1-s) F\left(\widetilde{K}, \frac{1}{\rho} L\right) .
$$

Considering that $F$ is a concave first-order homogeneous increasing on each variable function, we find that in the case of the constant rate of accumulation, the consumption is a concave increasing function of the labor force. Note that the specific consumption $\omega$ is expressed by the rate of accumulation $s$ through the equality

$$
\omega=\frac{C(L)}{L}=(1-s) F\left(\bar{\eta}, \frac{1}{\rho}\right),
$$

where $\bar{\eta}=\frac{\widetilde{K}}{\bar{L}}$ is a capital at the initial time $t$. Thus $s$ does not depend on $L$ if and only if when $\omega$ does not depend on $L$ (under the condition that growth rate $\rho$ is fixed).

It is natural to assume that per capita consumption $\omega$ depends on the total labor force. One way to consider such a relationship is suggested in [1]. It is interesting: does $\mathrm{C}(\mathrm{L})$ keep the properties which it has by constant $\omega$ (constant $s$ ) for the case, when 
$\omega$ depends on $L$ by given in [1] relationship? In this paper, we show that the answer to this question depends strongly on the type of production function. In particular, for the function with a constant elasticity of substitution (CES) consumption decreases for sufficiently large and convex $L$ and decreases and is concave (as a function of $L$ ), moreover $\lim _{L \rightarrow \infty} C(L)=0$.

In the second part we study the optimal distribution of labor between two single-product models in the case, when the optimality criterion is a total consumption. In this case, we essentially use the results of the first part.

In [1] the following way for the choice is proposed

$$
\omega=\frac{f(\eta)-\eta f^{\prime}(\eta)}{v+f^{\prime}(\eta)}
$$

where $\eta=\eta(L)$ is a root of the equation

$$
\eta=\frac{M}{L}-\frac{f(\eta)-\eta f^{\prime}(\eta)}{v+f^{\prime}(\eta)}
$$

Here $f(\eta)=F(\eta, 1), \eta>0$. It is assumed that the function $F$ is first-order positively homogeneous and $F(0, L)=F(K, 0)=0$. We also assume that the function $f$ is triple continuously differentiable, moreover, $f^{\prime}(\eta)>0, f^{\prime \prime}(\eta) \leq 0$ by $\eta>0$. Through $M$ is denoted the quantity $v \bar{K}+F(\bar{K}, \bar{L})$ - national wealth at the time $t$.

It will be convenient to consider sometimes the considered quantities as functions of functions function of the variable $\eta$. Define the following functions:

$$
\begin{aligned}
& \alpha(\eta)=v \eta+f(\eta) ; \beta(\eta)=v+f^{\prime}(\eta) \\
& \gamma(\eta)=f^{\prime \prime}(\eta) ; \delta(\eta)=f(\eta)-\eta f^{\prime}(\eta) .
\end{aligned}
$$

It is clear that

$$
\alpha^{\prime}=\beta, \beta^{\prime}=\gamma, \delta^{\prime}=-\eta \gamma .
$$

As follows from (1) the specific consumption $\omega$ has a form $\omega=\frac{\delta}{\beta}$. From the equation (2) one may express $L$ as a function of $\eta$ :

$$
L(\eta)=M \frac{\beta(\eta)}{\alpha(\eta)} .
$$

Note that $L$ is a decreasing function and

$$
\lim _{\eta \rightarrow+\infty} L(\eta)=0, \lim _{\eta \rightarrow+0} L(\eta)=+\infty .
$$

Thus

$$
C(L)=L \omega(L)=M \frac{\beta}{\alpha} \frac{\delta}{\beta}=M \frac{\delta(\eta(L))}{\alpha(\eta(L))},
$$

where $\eta(L)$ is an inverse to the function defined by the formula (5). From (4) and (5) follows that

$$
\eta^{\prime}(L)=\frac{1}{L^{\prime}(\eta)}=\frac{1}{M} \frac{\alpha^{2}}{\alpha \gamma-\beta^{2}} .
$$

Therefore

$C^{\prime}(L)=M\left(\frac{\delta}{\alpha}\right)^{\prime}=\frac{\delta^{\prime} \alpha-\alpha^{\prime} \delta}{\alpha^{2}} \eta^{\prime}(L)=\frac{\alpha^{\prime} \delta-\alpha \delta^{\prime}}{\beta^{2}-\alpha \gamma}$.

Since $\gamma(\eta)=f^{\prime \prime}(\eta)<0$, sign of the derivative $C^{\prime}(L)$ depends on the sign of the expression $\alpha^{\prime} \delta-\delta^{\prime} \alpha$ (calculated at a point $\left.\eta(\mathrm{L})\right)$. This, however, follows directly from (6).

After some simple calculations one may show that

$$
C^{\prime \prime}(L)=\frac{\left(\alpha^{\prime \prime} \delta-\delta^{\prime \prime} \alpha\right)\left(\beta^{2}-\alpha \gamma\right)-\left(\beta \gamma-\alpha \gamma^{\prime}\right) c^{\prime}}{\left(\beta^{2}-\alpha \gamma\right)^{2}} \eta^{\prime},
$$

from which the following equality is obtained

$$
C^{\prime \prime}(L)=\frac{\gamma\left(\beta^{2}-2 \alpha \gamma\right)+\alpha \beta \gamma^{\prime}}{\left(\beta^{2}-\alpha \gamma\right)^{2}} .
$$

Considering that $\alpha>0, \eta^{\prime}<0$, we have

$$
\operatorname{sign} C^{\prime \prime}(L)=-\operatorname{sign}\left[\alpha \gamma^{\prime} \beta-2 \alpha \gamma^{2}+\gamma \beta^{2}\right] \text {. }
$$

Note that the sign of $C^{\prime \prime}(L)$ depends on the third derivative of the function $f$. If $\gamma^{\prime}(\eta)=f^{\prime \prime \prime}(\eta)<0$, then $C^{\prime \prime}(L)>0$. If $\gamma^{\prime}(\eta)>0$, there is nothing certain about the sign of $C^{\prime \prime}(L)$. 
We consider separately the cases when $F$ is Cobb-Douglas function and function with constant elasticity of substitution (CES).

Theorem 1. Let $F(K . L)=A K^{r} L^{1-r}$ be the Cobb-Douglas function (here $0<r<1$ ), and per capita consumption is calculated using the formulas (1), (2).

Then $C(L)$ is increasing concave function such that $\lim _{L \rightarrow \infty} C(L)<+\infty$.

Proof. As follows from (6), consumption is a decreasing function of $\alpha / \delta$. Compute the function $\alpha / \delta$. Then we get

$$
\frac{\alpha(\eta)}{\delta(\eta)}=\frac{v \eta+A \eta^{r}}{A(1-r) \eta^{2}}=\frac{1}{A(1-r)}\left[v \eta^{1-r}+A\right]
$$

which implies that $\frac{\alpha(\eta)}{\delta(\eta)}$ is an increasing function of the variable $\eta$. Since the function $\eta(L)$ is decreasing, the function $C(L)$ increases. To determine the sign of the second derivative of the function $C$ we use (10). It is easy to check that in this case $\alpha \beta \gamma^{\prime}+\beta^{2} \gamma-2 \alpha \gamma^{2}>0$ for all, and therefore $C^{\prime \prime}(L)<0$ for all $L$. As follows from (6) and (11)

$$
\lim _{L \rightarrow \infty} C(L)=\lim _{\eta \rightarrow+0} M \frac{\delta(\eta)}{\alpha(\eta)}=M(1-r)<+\infty .
$$

The theorem is proved.

Let $F(K, L)=\left(A K^{-\rho}+B L^{-\rho}\right)^{-\frac{1}{\rho}}$ be a function with constant elasticity of substitution. We consider here the most interesting case $\rho>1$ (investigation of cases $\rho \in(0,1]$ and $\rho \in(-1,0]$ follows in the same pattern as below). We introduce the notation $Y=A+B \eta^{\rho}$. Then the function $f(\eta)=F(\eta, 1)$ and its derivatives take the form:

$$
\begin{gathered}
f(\eta)=\eta Y^{-\frac{1}{\rho}} ; \\
f^{\prime}(\eta)=A Y^{-\frac{1}{\rho}-1} ; \\
f^{\prime \prime}(\eta)=-(\rho+1) A B \eta^{\rho-1} y^{-\frac{1}{\rho}-2} ;
\end{gathered}
$$

$$
\begin{aligned}
& f^{\prime \prime \prime}(\eta)= \\
& -(\rho+1) A B \eta^{\rho-2} Y^{-\frac{1}{\rho}-3}\left(A(\rho-1)-(2+\rho) B \eta^{\rho}\right) .
\end{aligned}
$$

Substituting the values $f, f^{\prime}, f^{\prime \prime}$ into the formulas (7), we see that

$$
C^{\prime}(L)=Z\left(v B \eta^{\rho}-v A \rho-A \rho Y^{-\frac{1}{\rho}}\right),
$$

where $Z>0$. Thus

$$
\operatorname{sign} C^{\prime}(L)=\operatorname{sign}\left(v B \eta^{\rho}-v A \rho-A \rho Y^{-\frac{1}{\rho}}\right) \text {. }
$$

Here, as above, $\eta=\eta(L)$ is a function of the inverse of the function $L(\eta)$ defined by the formula

(5). Take $g(\eta)=v B \eta^{\rho}-v A \rho-A \rho\left(A+B \eta^{\rho}\right)^{-\frac{1}{\rho}}$.

Lemma 1. The equation $g(\eta)=0$ has a unique solution $\bar{\eta}_{1}$, on the positive axis and $g(\eta)>0$ by

Proof. It is easy to verify that $g^{\prime}(\eta)>0$, and the function $g$ is therefore increasing. Take $x=\eta^{\rho}$. Since the graphs of functions $g_{1}(x)=v B x-v A \rho$ and $g_{2}(x)=A \rho(A+B x)^{-\frac{1}{\rho}}$ intersect, then the equation $g(\eta)=0$ has a solution.

The lemma is proved.

Lemma 2. let $\eta \geq \bar{\eta}_{1}$, where $\bar{\eta}_{1}$ is a root of the equation $g(\eta)=0$, the functions $\alpha$ and $\delta$ are defined by the formulas (3), $f(\eta)=\eta Y^{-\frac{1}{\rho}}$, where $Y+A+B \eta^{\rho} S$. Then

$$
\alpha^{\prime \prime}(\eta) \delta(\eta)-\delta^{\prime \prime}(\eta) \alpha(\eta) \geq 0 .
$$

Proof. Let $\gamma(\eta)=f^{\prime \prime}(\eta)$. Using the formulas (12) и (13), it is easy to show that

$$
\gamma^{\prime}=\gamma \frac{A(\rho-1)-B \eta^{\rho}(2+\rho)}{\eta Y} .
$$

Let us transfer the expression $\alpha^{\prime \prime} \delta-\delta^{\prime} \alpha$

$$
\begin{aligned}
& \alpha^{\prime \prime} \delta-\delta^{\prime \prime} \alpha=\gamma(\alpha+\delta)+\eta \alpha \gamma^{\prime}= \\
& =\frac{\gamma}{Y}\left[\alpha Y+\delta Y+\alpha\left(A(\rho-1)-(2+\rho) B \eta^{\rho}\right)\right] .
\end{aligned}
$$


After some simplifications, it can be shown that the expression in square brackets can be written as follows:

$\eta\left[\left(v A \rho-v B \eta \rho+A \rho Y^{-\frac{1}{\rho}}\right)-B \eta^{\rho} \rho\left(Y^{-\frac{1}{\rho}}+Y\right)\right]$.

By Lemma 1, the first term in square brackets is negative. To complete the proof it is sufficient to consider that $\gamma<0$.

\section{Theorem}

2

Suppose

that

$F(K, L)=\left(A K^{-\rho}+B L^{-\rho}\right)^{-\frac{1}{\rho}}$, where per capita consumption $\omega$ is calculated using the formula (1), (2). Then: 1) Consumption $C(L)$ reaches its maximum on the positive half-axis at a certain point $\bar{L}_{1}$, and this is the only local extremum point; 2) The function $C(L)$ has a unique inflection point at which the concavity changes to convexity, and $L_{2}>L_{1}$; 3) $\operatorname{Lim}_{L \rightarrow \infty} C(L)=0$

Proof. Let $\overline{\eta_{1}}$ is a root of the existence of which is guaranteed by Lemma 1 , and $\overline{L_{1}}=L\left(\bar{\eta}_{1}\right)$. Then, as follows from (14) 1 and monotone decreasing of the function $\eta(L)$ in the interval $\left(0, \bar{L}_{1}\right)$, consumption $C(L)$ increases, and in the $\left(\bar{L}_{1},+\infty\right)$ decreases. Thus, the first part of the theorem is proved.

Take

$$
\tilde{\eta}=\left(\frac{A(\rho-1)}{B(2+\rho)}\right)^{\frac{1}{\rho}} ; \quad \widetilde{L}=L(\widetilde{\eta}) .
$$

Since by the condition of the theorem $\rho>1$, so as follows from (13), $f^{\prime \prime \prime}(\eta)<0$ by $\eta<\bar{\eta}$ and $f^{\prime \prime \prime}>0$ by $\eta>\tilde{\eta}$. Using the definition of the point $\widetilde{\eta}$, it is easy to verify that

$$
v B \widetilde{\eta}^{\rho}-v A \rho-A \rho(A+B \tilde{\eta})^{-\frac{1}{\rho}}<0,
$$

Therefore as follows from (14), $C^{\prime}(\bar{L})<0$. Considering part 1 of the theorem we see the validity of the inequality $\widetilde{L}>L_{1}$.
Let $L \geq \widetilde{L}$. Then, $\eta=\eta(L) \leq \widetilde{\eta}$, and hence $f^{\prime \prime \prime}(\eta) \leq 0$. In this case, as follows from (10), the inequality $C^{\prime \prime}(L)<0$ is true. Now we show that the inequality $C^{\prime \prime}(L)>0$ is valid in the interval $\left(0, \bar{L}_{1}\right)$. Indeed, since in this interval $\gamma^{\prime \prime}=f^{\prime \prime \prime}(\eta(L)) \geq 0$ and at the same time $C^{\prime}(L)>0$, considering Lemma 2 and formula 8, we verify the validity of the required inequality.

From the above it follows that the continuous function $C^{\prime \prime}$ takes on the ends of the interval $\left(\bar{L}_{1}, L\right)$ the values of different signs, so it has a root $\bar{L}_{2}$ in this interval. From (10) it follows that all the roots of $C^{\prime \prime}$ coincide with the roots of the function $h(\eta(L))$, where

$$
h=\alpha \gamma^{\prime} \beta+\beta^{2} \gamma-2 \alpha \gamma^{2},
$$

$\alpha, \beta, \gamma$ are defined by the formula (3). Using the formula (15), let us transform $h$ as

$$
h(\eta)=\left(-v B \eta^{\rho}+v A \rho+A \rho Y^{-\frac{1}{\rho}}\right)
$$

$$
\left(1+v A Y^{\frac{1-\rho}{\rho}}\right)-v(1+\rho) B^{2} \eta^{2 \rho}\left(1+v Y^{\frac{1}{\rho}}\right) Y^{-1} .
$$

In the considered interval $\left(\overline{L_{1}}, \widetilde{L}\right)$, the inequality $C^{\prime}(L)<0$ holds, therefore, using (14) we can verify that the function $h(\eta(L))$ is monotonic. Thus, $\bar{L}_{2}$ is the only root of the function $C^{\prime \prime}$ on the interval $\left(\overline{L_{1}}, \widetilde{L}\right)$, and therefore, on the entire domain.

Compute $\lim _{L \rightarrow+\infty} C(L)$. We have, using (6),

$$
C(L)=M \frac{\delta(\eta)}{\alpha(\eta)}
$$

Where $\eta=\eta(L)$. In the considered case

$$
\begin{gathered}
\frac{\delta(\eta)}{\alpha(\eta)}=\frac{f(\eta)-\eta f^{\prime}(\eta)}{v \eta+f(\eta)} \\
v\left(A+B \eta^{\rho}\right)^{1+\frac{1}{\rho}}+\left(A+B \eta^{\rho}\right)
\end{gathered},
$$

Therefore, $\lim _{L \rightarrow+\infty} C(L)=\lim _{\eta \rightarrow 0} C(\eta)=0$. 
Theorem is proved.

Theorem 2 states that for the considered production function of consumption as a function of the labor force $L$ decreases with an increase $L$ starting from a certain threshold size $\bar{L}_{1}$, and becomes arbitrarily small for sufficiently large $L$. This seemingly paradoxical phenomenon can be explained as follows: if the accumulated national wealth $M$ is disproportionately small compared to the size of the labor force $L$, it is necessary first of all to increase this wealth through increased investments $I$ and thereby reduce consumption $C$. Moreover the greater $L$, or what is the same, the smaller the ratio $\frac{M}{L}$ (this appears in the basic equation (2)), the greater part of the production should be referred to increase the national wealth.
We give here two notations to the theorem 1 .

Note 1 . For a fixed $\rho$ maximum point $\bar{L}$ for the function $C(L)$ indeed is a function of the coefficients $A$ and $B$. It is not difficult to show that any number $L>0$ may join as a $\bar{L}_{1}$ by suitable choice of these coefficients.

Note 2. Can be verified that when $\rho \in(0,1]$ the function $C(L)$ is also, as in the case of $\rho>1$, only one maximum point and a single point of inflection. If $\rho \in(-1,0)$, we can, $C(L)$ just, is an increasing concave function, as in the case of the Cobb-Douglas function.

\section{References}

[1] Rubinov A.M. Mathematical Models of Expanded Reproduction. L., Nauka, 2003, 186 p. 